CARTA AL EDITOR

\section{ROL DEL ULTRASONIDO PULMONAR EN EL DIAGNÓSTICO DE LA NEUMONÍA POR SARS-COV-2: ¿ALTERNATIVA A LA ESCASEZ DE TOMÓGRAFOS?}

\section{ROLE OF PULMONARY ULTRASOUND IN THE DIAGNOSIS OF SARS-COV-2 PNEUMONIA: AN ALTERNATIVE TO THE SCARCITY OF CT SCANNERS?}

\section{Samuel Pecho-Silva (iD) 1,2,a \\ ${ }^{1}$ Universidad Científica del Sur, Lima, Perú. \\ ${ }^{2}$ Hospital Nacional Edgardo Rebagliati Martins, Lima, Perú. \\ ${ }^{a}$ Médico cirujano, especialista en Neumología.}

Sr. editor: El ultrasonido pulmonar (UP) es una herramienta diagnóstica importante para el médico y su uso se extiende a las unidades de medicina crítica. Se ha descrito que el UP puede emplearse en la detección de alteraciones, como consolidaciones, efusiones pleurales, neumotórax; y en la evaluación de la vía aérea superior y enfermedades como, los síndromes intersticiales, el síndrome de dificultad respiratoria aguda, entre otras ${ }^{(1)}$. Por estas razones, el UP es uno de los primeros procedimientos que se debe realizar en caso de patología pulmonar aguda.

Algunas instituciones, como la Sociedad Española de Neumología y Cirugía Torácica, Sociedad Española de Endoscopía Respiratoria, Intensive Care Society, The Society for Acute Medicine, World Interactive Network Focused on Critical Ultrasound y World Federation for Ultrasound in Medicine and Biology, coinciden en que el UP tiene potencial utilidad en el diagnóstico precoz de la neumonía por SARS-CoV-2, su monitorización, seguimiento clínico y evaluación de la respuesta al tratamiento. Además, es útil en las unidades de cuidados intensivos como ayuda en la decisión de «destete» del ventilador mecánico ${ }^{(2)}$. Asimismo, disminuye la exposición de los profesionales de la salud a la COVID-19, ya que un solo médico puede realizar toda la evaluación de manera objetiva al lado de la cama del paciente ${ }^{(2)}$.

Por otro lado, la tomografía de tórax (TC) es fundamental para el diagnóstico precoz de las lesiones pulmonares producidas

Citar como: Pecho-Silva S. Rol del ultrasonido pulmonar en el diagnóstico de la neumonía por SARS-CoV-2: ¿Alternativa a la escasez de tomógrafos? Rev Peru Med Exp Salud Publica. 2020;37(2):381-2. doi: https://doi.org/10.17843/ rpmesp.2020.372.5506

Correspondencia: Samuel Pecho Silva; Jirón Moore 228, Magdalena del Mar Lima, Perú; samuelpechosilva@gmail.com

Recibido: 06/04/2020 Aprobado: 29/04/2020 En línea: 03/05/2020 por la COVID-19 y ha demostrado una mayor sensibilidad $(97,2 \%)$ que la prueba molecular de reacción en cadena de la polimerasa con transcriptasa reversa (RT-PCR, por sus siglas en inglés) $(83,3 \%)^{(3)}$. Sin embargo, la poca disponibilidad de la TC en los establecimientos de salud es un obstáculo para su uso masivo, incluso, en aquellos lugares donde está disponible, su uso se ha limitado por el temor a la contaminación del equipo.

Los principales hallazgos en el UP en pacientes con COVID-19 son las lesiones de disposición basal, posteriores y subpleurales con un patrón difuso y multifocal de «líneas $\mathrm{B}$ » $\mathrm{y}$ con una línea pleural engrosada e irregular. Las consolidaciones suelen ser subpleurales, multifocales y lobares con broncograma aéreo dinámico con escaso suministro sanguíneo. De estos hallazgos, el más frecuente es la presencia de «líneas B» que son fácilmente evaluables y reconocibles con el UP ${ }^{(4)}$.

Se realizó una búsqueda en PubMed de artículos publicados del 2 al 25 de abril de 2020 con el objetivo de determinar la validez del UP en pacientes con COVID-19 y complementar la revisión realizada por Smith et al. ${ }^{(4)}$. Se utilizó la siguiente estrategia de búsqueda: lung OR chest OR pulmonary OR pul$\mathrm{mo}^{*}$ OR thorax OR thora* and ultrasound OR sonography OR ultraso* OR sonog* AND COVID* OR SAR-CoV-2. Se encontraron cinco artículos (cartas al editor, reporte de caso y series de casos) que incluyeron a 77 pacientes adicionales (cinco gestantes) a los 54 encontrados por Smith et al. ${ }^{(4)}$. Todos ellos con diagnóstico confirmado de COVID-19 por RT-PCR ${ }^{(5,6)}$. Solo Lu et al. ${ }^{(6)}$ informó la sensibilidad, especificidad y precisión diagnóstica del UP. No fue posible calcular la validez del UP a partir de los reportes del resto de autores. En la Tabla 1 se muestran los resultados y se compara la sensibilidad del UP ${ }^{(6)}$ con la de la TC y la de la RT-PCR ${ }^{(3)}$.

Las ventajas adicionales del UP frente a otros métodos diagnósticos son su amplia disponibilidad en establecimientos de salud, bajo costo, facilidad de acceso, rápida movilización y limpieza, fácil realización y posibilidad de evaluación dinámica del paciente con este procedimiento. A diferencia de la TC, el UP es un procedimiento diagnóstico seguro en gestantes y las imágenes ecográficas pueden ser fácilmente usadas en telemedicina. La única limitación del UP es que no permite detectar lesiones que no sean subpleurales, sin embargo, esto sería poco relevante ya que las lesiones por COVID-19 suelen serlo.

En tal sentido, es recomendable el uso del UP en los pacientes con sospecha y diagnóstico de COVID-19 que sean atendidos en un establecimiento que no cuente con la TC. De igual modo, en aquellos que cuenten con TC, el UP servirá para el seguimiento clínico y como criterio adicional para definir el alta hospitalaria.

Contribuciones de autoría: El autor concibió, diseñó, redactó y aprobó la versión final del artículo. 
Tabla 1. Sensibilidad, especificidad y precisión diagnóstica del ultrasonido pulmonar comparado con la tomografía de tórax y la RT-PCR al momento del diagnóstico inicial de COVID-19

\begin{tabular}{lccccc}
\hline $\begin{array}{l}\text { Autor } \\
\text { (país) }\end{array}$ & Método & Gravedad & $\begin{array}{c}\text { Sensibilidad } \\
\mathbf{n} / \mathbf{N}(\%)\end{array}$ & $\begin{array}{c}\text { Especificidad } \\
\mathbf{n} / \mathbf{N}(\%)\end{array}$ & $\begin{array}{c}\text { Precisión diagnóstica } \\
\mathbf{n} / \mathbf{N}(\%)\end{array}$ \\
\hline & UP & Leve & $11 / 16(68,8)$ & $12 / 14(85,7)$ & $23 / 30(76,7)$ \\
Lu et al. & & Moderada & $7 / 9(77,8)$ & $16 / 21(76,2)$ & $23 / 30(76,7)$ \\
$(\text { China })^{(6)}$ & & Grave & $2 / 2(100)$ & $26 / 28(92,9)$ & $28 / 30(93,3)$ \\
& TC & Grave & NR & NR \\
Long et al. $_{(\text {China })^{(3)}}$ & RT-PCR & Grave $^{\mathrm{a}}$ & $35 / 36(97,2)^{\mathrm{b}}$ & $\mathrm{NR}$ & \\
\hline
\end{tabular}

${ }^{a}$ Basado en las características clínicas descritas por los autores. ${ }^{\mathrm{b}}$ Calculado al momento del diagnóstico inicial.

RT-PCR: reacción en cadena de la polimerasa con transcriptasa reversa; UP: ultrasonido pulmonar; TC: tomografia de tórax; NR: no reportado.

Fuentes de financiamiento: Autofinanciado.

Declaración de conflictos de interés: El autor declara que no tiene conflicto de interés alguno.

\section{REFERENCIAS BIBLIOGRÁFICAS}

1. Kristensen MS, Teoh WH, Graumann O, Laursen CB. Ultrasonography for clinical decision-making and intervention in airway management: From the mouth to the lungs and pleurae. Insights Imaging. 2014;5(2):253-79. doi: 10.1007/s13244-014-0309-5.

2. Soldati G, Smargiassi A, Inchingolo R, Buonsenso D, Perrone T, Federica $\mathrm{D}$, et al. Is there a role for lung ultrasound during the COVID-19 pandemic?. J Ultrasound Med. 2020. doi: 10.1002/jum.15284.
3. Long C, Xu H, Shen Q, Zhang X, Fan B, Wang C, et al. Diagnosis of the Coronavirus Disease (COVID-19): rRT-PCR or CT?. Eur J Radiol. 2020;126:108961. doi: 10.1016/j.ejrad.2020.108961.

4. Smith MJ, Hayward SA, Innes SM, Miller A. Point-of-care lung ultrasound in patients with COVID-19 - a narrative review. Anaesthesia. 2020. doi: https://doi.org/10.1111/anae.15082.

5. Peng QY, Wang XT, Zhang LN, and Chinese Critical Care Ultrasound Study Group (CCUSG). Findings of lung ultrasonography of novel corona virus pneumonia during the 2019 - 2020 epidemic. Intensive Care Med. 2020;(87):6-7. doi: 10.1007/s00134-020-05996-6.

6. Lu W, Zhang S, Chen B, Jiaxin C, Xian J, Lin Y, et al. A Clinical Study of Noninvasive Assessment of Lung Lesions in Patients with Coronavirus Disease-19 (COVID-19) by Bedside Ultrasound. Eur J Ultrasound. 2020. doi: https://doi.org/10.1055/a-1154-8795. 